\title{
Efficacy of pentasodium diethylenetriamine pentaacetate in ameliorating anosmia post COVD-19
}

\author{
Mohamed hussien ${ }^{1}$, Ahmed Hussien ${ }^{1}$, Wael ismail ${ }^{1}$, Mahmoud alsoubky ${ }^{1}$, sherief ramzy ${ }^{1}$, \\ and Mohamed Shahin ${ }^{2}$ \\ ${ }^{1} \mathrm{Al}-$ Azhar University \\ ${ }^{2}$ Damanhour University
}

February 28, 2022

\begin{abstract}
Abstract Objectives: An association between COVID-19 and anosmia has been demonstrated worldwide. Calcium cations play an essential role in odor transmission, including feedback inhibition. Therefore, it is suggested that reducing intranasal free calcium cations with topical chelating agent pentasodium diethylenetriamine pentaacetate may improve olfactory function in patients with anosmia post COVID-19 infection. Design: Prospective controlled clinical trial to test use of pentasodium diethylenetriamine pentaacetate for post COVID-19 olfactory loss. Setting: ENT Department (Institution blinded for review). Participants: Sixty-six adult patients with history of confirmed COVID-19 and olfactory dysfunction persisted more than 90 days after SARS-CoV-2 negative testing were included. Main outcome measures: Participants were divided into 2 equal groups receiving nasal spray containing either $0.9 \%$ sodium chloride or $2 \%$ pentasodium diethylenetriamine pentaacetate. Olfactory function was assessed before treatment and 1 month later using the Sniffin' Sticks test. A carbon paste ion-selective electrode was developed for quantitative analysis of calcium cation concentrations in nasal mucus before treatment and 1 month later. Results: After treatment with pentasodium diethylenetriamine pentaacetate, there was a significant improvement from functional anosmia to hyposmia compared with sodium chloride. In addition, the decrease of calcium concentration was recorded after treatment with pentasodium diethylenetriamine pentaacetate compared with sodium chloride. Conclusion: Based on the results of the proposed study, topical use of pentasodium diethylenetriamine pentaacetate may be a useful therapy for olfactory dysfunction after COVID-19.
\end{abstract}

\section{Efficacy of pentasodium diethylenetriamine pentaacetate in ameliorating anosmia post COVD- 19}

Running title: Pentasodium pentetate in COVID-19

\begin{abstract}
Objectives: An association between COVID-19 and anosmia has been demonstrated worldwide. Calcium cations play an essential role in odor transmission, including feedback inhibition. Therefore, it is suggested that reducing intranasal free calcium cations with topical chelating agent pentasodium diethylenetriamine pentaacetate may improve olfactory function in patients with anosmia post COVID-19 infection.
\end{abstract}

Design: Prospective controlled clinical trial to test use of pentasodium diethylenetriamine pentaacetate for post COVID-19 olfactory loss.

Setting: ENT Department (Institution blinded for review).

Participants : Sixty-six adult patients with history of confirmed COVID-19 and olfactory dysfunction persisted more than 90 days after SARS-CoV-2 negative testing were included. 
Main outcome measures: Participants were divided into 2 equal groups receiving nasal spray containing either $0.9 \%$ sodium chloride or $2 \%$ pentasodium diethylenetriamine pentaacetate. Olfactory function was assessed before treatment and 1 month later using the Sniffin' Sticks test. A carbon paste ion-selective electrode was developed for quantitative analysis of calcium cation concentrations in nasal mucus before treatment and 1 month later.

Results: After treatment with pentasodium diethylenetriamine pentaacetate, there was a significant improvement from functional anosmia to hyposmia compared with sodium chloride. In addition, the decrease of calcium concentration was recorded after treatment with pentasodium diethylenetriamine pentaacetate compared with sodium chloride.

Conclusion: Based on the results of the proposed study, topical use of pentasodium diethylenetriamine pentaacetate may be a useful therapy for olfactory dysfunction after COVID-19.

Keywords : Anosmia; olfactory test; COVID-19.

\section{Key points:}

- Reports demonstrated an association between COVID-19 and olfactory dysfunction

- Olfactory dysfunction causes an increase in intracellular calcium concentration

- Pentasodium diethylenetriamine pentaacetate has ability to decrease calcium concentration in the nasal secretion

- Intranasal pentasodium diethylenetriamine pentaacetate may be a useful therapy for olfactory dysfunction

- Significant improvement was observed from functional anosmia to hyposmia

\section{Introduction}

Anosmia is the inability to smell which may be partial or total. Recently, anosmia considered a characteristic sign that helps in the diagnosis of COVID-19. The exact underlying pathogenesis is not well understood 1. Different studies investigated the relationship between olfactory dysfunction post COVID-19 and the recovery rate of loss of smell. In the first 2 months, it was reported that $79.5 \%$ of patients may expect a complete recovery of the olfactory function. Also $20.5 \%$ of patients could not achieve normal levels of olfactory function ${ }^{2}$.

The nasal cavity is the first gateway for the initial infection as viral loads in the patient's nasal cavity are higher than the viral loads in the pharynx. Also, the nasal cavity environment plays an important role in the process of olfaction transmission from the nasal cavity to the central processing system ${ }^{3}$.

Calcium cation has been shown to play an essential role in the mechanism of smell transmission through inhibitory feedback inhibition approach. This inhibitory role is currently thought to occur through two distinct mechanisms ${ }^{4}$. First, calcium calmodulin interacts directly with the cyclic nucleotide-gated channels, resulting in decreased sensitivity of the channel to cyclic nucleotides and thus decreased positive current influx. Second, calcium-dependent phosphorylation of adenylyl cyclase results in decreased production of intracellular cyclic adenosine monophosphate and thus decreased activation of cation channels controlled by cyclic nucleotides. Accordingly, it has been suggested that reducing intranasal free calcium may improve olfactory function in patients with anosmia related COVID- $19^{5}$.

Pentasodium diethylenetriamine pentaacetate (DTPA) is a potential chelating agent used for complexation and sequestration of metal ions. It has the ability to chelate divalent and trivalent metal ions. DTPA is used in a variety of cosmetic products to remove calcium and magnesium cations that interfere with foaming and cleansing performance and that can cause turbidity in clear liquids ${ }^{6,7}$. DTPA has a high chelating capacity for calcium cations in an alkaline $\mathrm{pH}$ medium and forms a stable complex product, suggesting that reducing intranasal free calcium may improve anosmia in patients post COVID-19 infection.

The main objective of this work is to test the effect of topical DTPA on reducing the increase of calcium cations in the mucus of patients with anosmia related COVID-19. This is the first published clinical trial 
testing DTPA as a topical treatment to improve anosmia post COVID-19 infection.

\section{Materials and methods}

Ethics

Informed consent was obtained before participation in the study, which represented standard practice of care at the time in accordance with the practice guidelines of the (blinded for review). This study was approved by the Ethical Committee of (blinded for review) under trial number IRB00012367-20-09-008.

Study design and setting

Prospective randomized controlled trial study was conducted at the ENT Department of (blinded for review). The study design in accordance with reporting guidelines was shown in Figure 1.

Participants

Sixty-six anosmia related COVID -19 adult patients, 36 females and 30 males, were recruited during a period from August 2020 to January 2021 for this prospective trial study. Patients provided written informed consent for their medical records before participating in this study. Only patients over the age of 18 with history of confirmed COVID-19 and olfactory dysfunction persisted more than 90 days after SARS-CoV-2 negative testing were included. Patients with neurodegenerative or other conditions affecting olfaction were excluded. The patient characteristics were recorded for each participant.

Treatment regimen

Patients were divided into 2 equal groups: intranasal $0.9 \%$ sodium chloride (group A) and intranasal $2 \%$ DTPA in borate buffer, pH 8 (group B). The pharmaceutical preparations of the described treatment were prepared by (blinded for review). These preparations were filled into identical opaque nasal spray bottles delivering a volume of $0.1 \mathrm{~mL}$. The prescribed treatment bottle was administered to the participants 3 times daily for 1 month. The sodium chloride group was used to eliminate the variations between the treated and non-treated groups. Improvement in the olfactory function was demonstrated. In addition, participants' nasal secretions were collected before treatment and 1 month later. The concentrations of calcium cations of the collected nasal secretions were determined using an ion-selective electrode.

Main outcome measures

Burghart Sniffin' Sticks(R, a widely assessment test for smell disorders ${ }^{8}$, was used to assess the olfactory function in the study participants. Four values, threshold (T), discrimination (D), identification (I) and the TDI values were measured before treatment and 1 month later. A TDI value below 16.75 points represents functional anosmia. Also TDI value between 16.75 and 30.50 points represents hyposmia and TDI value of 30.75 points or more represents normosmia. The patient is considered to be improved when the TDI value has increased by 6 points

Participants' nasal secretion was collected before treatment and 1 month later. Collection occurred immediately after sneezing. A stainless steel (approximately $10 \mathrm{~mm} \times 5 \mathrm{~mm} \times 2 \mathrm{~mm}$ ) was clamped on the septum between the nostrils to allow the mucus to drain into a special $1.5-\mathrm{mL}$ tube ${ }^{9}$. The collected secretion was transferred to centrifugation tubes after adding $0.5 \mathrm{~mL}$ of borate buffer solution. The protein content of the mucus was denatured by adding $3 \mathrm{~mL}$ borate buffer solution. The collected tubes were centrifuged at 4000 rpm for 30 minutes. The protein-free supernatant was evaporated and the residues obtained were dissolved in $10 \mathrm{~mL}$ of borate buffer solution. The carbon paste ion-selective electrode ${ }^{10}$ was prepared by (blinded for review). It was designed to quantitatively analyze the calcium cation concentration of the collected nasal secretions of the participants before treatment and 1 month later.

Statistical analysis

All statistical analyses were performed using SPSS v23 statistical software (SPSS, Inc, Chicago, Illinois). Sample size was tested using Fisher's exact probability test. Student's $t$-tests, paired and unpaired $t$-tests, 
were used to test the significance of the data obtained. Statistical significance was assigned when $p<0.05$. In general, results were reported as mean \pm standard deviation unless otherwise stated.

\section{Results}

Sixty-six adult patients with history of confirmed COVID-19 and olfactory dysfunction persisted more than 90 days after SARS-CoV-2 negative testing were enrolled in this prospective study. The age of the patients ranged from 18 to 55 years. There were 38 females and 28 males. The complete characteristics of the patients were described in Table 1. Fisher's exact probability test was used to assess the sample size. Non-significant difference in the frequency of smoking between the sodium chloride group and the DTPA group (5/28 vs. $4 / 29 ; p=1.00)$. Differences in the frequency of comorbidities were also assessed and the results were not significant between the sodium chloride group and the DTPA group, as shown in Table 1.

Intra-nasal administration of DTPA had the ability to form a calcium-DTPA complex and decreased the concentrations of calcium cations in the mucus of the participants. DTPA had the selective ability to sequester calcium cations at an optimized $\mathrm{pH}$ of 8 and form stable complex products. The schematic reaction pathway for the reaction of DTPA with calcium cations was shown in Figure 2.

Olfactory performance was evaluated using the Sniffin' Sticks test before and after treatment with topical sodium chloride or DTPA. T, D, I and TDI were measured in each participant before treatment and 1 month later. Mean T, D, I and TDI values before and after topical sodium chloride and DTPA treatment for patients were shown in Table 2. The TDI values were compared with the reported reference values. The mean values of the TDI scores were calculated and presented in Table 2. Regarding to the olfactory assessed score of DTPA group, 27 patients (84.38\%) showed an improved olfactory function and 5 patients $(15.63 \%)$ did not show improvement. This may be due to topical nasal sprays generally have quite variable compliance and delivery efficiency, especially to the olfactory cleft, so one would expect some subjects with little to no response even to a highly efficacious drug. The results showed that the mean \pm SD of the TDI value before and receiving sodium chloride was $10.87 \pm 1.24$ and $10.93 \pm 1.09$, respectively, Table 2 . While, the mean \pm SD of the TDI value before and after receiving DTPA was $11.75 \pm 1.22$ and $20.39 \pm 3.25$, respectively, Table 2. The results indicated non-significant improvement in patients receiving sodium chloride. On the other hand, there was a significant improvement in patients receiving DTPA with clinical change from functional anosmia to hyposmia.

The concentration of calcium cations was quantitatively determined in the nasal mucus of all patients. A selective electrode made of carbon paste was developed for the electrochemical determination of calcium cations. First, the values of electromotive force were measured in a calcium concentration range of 100 to $0.001 \mathrm{mM}$ to obtain a calibration diagram relating the values of electromotive force to the negative logarithmic value of calcium cation concentration. The designed sensor exhibited a near Nernstian slope of $28.25 \mathrm{mV} /$ decade with a detection limit of $0.0001 \mathrm{mM}$ in a linear range of 100 to $0.001 \mathrm{mM}$ calcium cation concentration. The concentrations of calcium cations in nasal secretions were successfully determined using the developed electrode. The mean \pm SD of calcium cation concentration $(\mathrm{mM})$ before and after receiving sodium chloride was $38.39 \pm 3.04$ and $37.06 \pm 2.64$, respectively, Table 2. Also, the mean \pm SD of calcium concentration (mM) before and after receiving DTPA was $37.54 \pm 2.06$ and $24.39 \pm 4.52$, respectively, Table 2 . The results showed a significant decrease in calcium concentration in the patients received DTPA.

Statistical analysis, paired $t$ test, was used to compare the means and standard deviations of the TDI values for the sodium chloride or DTPA groups before and after treatment. Comparison of the results for the DTPA group revealed a significant difference with a relevant clinical improvement in olfactory performance, $t(32)=$ $16.60, p<0$.00001. Similarly, comparison of the results for the sodium chloride group revealed non-significant difference without clinical improvement in olfactory performance, $t(32)=0.42, p=0.338$. Also, an unpaired $t$ test was used to compare mean TDI values after receiving DTPA with TDI values after receiving sodium chloride. The significant difference was indicated, $t(39)=15.76, p<0.00001$.

\section{Discussion}


Olfactory dysfunction has become one of the common symptoms associated with many cases of corona virus

11-13. During the response to odorants, there is a simultaneous increase in cyclic adenosine monophosphate and calcium cations. The cyclic adenosine monophosphate is produced by activation of adenylate cyclase and the increase in calcium cations is due to influx via cyclic nucleotide channels. Therefore, it is hypothesized that decreasing intranasal free calcium cations may reduce feedback inhibition and lead to improved receptor response and olfactory function ${ }^{14}$.

This prospective randomized controlled trial tested the topical application of DTPA for the treatment of anosmia post COVID-19 infection. DTPA is a chelating agent that can strongly sequester divalent and trivalent cations and form a strong metal complex product. DTPA wraps around a metal ion forming up to eight bonds. At pH 8, DTPA selectively forms a calcium-DTPA complex even in the presence of sodium, potassium or magnesium cations. This reaction mechanism leads to a decrease in calcium cation in the nasal secretions. The described results confirm that the reduction of calcium cations in by DTPA improves the olfactory performance.

The "Sniffin' Sticks" test is a widely used olfactory performance assessment tool consisting of three subtests: odor threshold, odor discrimination and odor identification. It was introduced over 20 years ago and its test-retest reliability and validity have been demonstrated. The calculated scores of the odor test confirmed that topical application of DTPA produces significant improvement in odor performance ${ }^{15,16}$.

Quantitative analysis of calcium cations provides an indication of the increase or decrease in calcium cations. This approach is based on the design of a carbon paste ion-selective electrode. Carbon paste electrodes are considered as an important type of ion-selective electrodes due to their advantages of chemical inertness, robustness, stable response, no need for internal solution and suitability for a variety of sensing and detection applications. Also, it can be used with a small volume of nasal secretion ${ }^{10}$. Using the developed electrode, it was possible to determine the calcium cation concentration before and after the described treatment. The results obtained showed a sharp decrease in calcium cation concentration in the patients treated with topical DTPA. This can be explained by the chelation of the calcium cation and the formation of the calcium DTPA complex product.

This paper introduced an evidence for a relationship between lowering calcium cation concentration in the nasal secretions by DTPA and the improvement in the olfactory performance.

\section{Conclusion}

This paper demonstrated the effect of topical pentasodium diethylenetriamine pentaacetate in lowering an elevated calcium cation in mucus of anosmia-related COVD-19 infection. After treatment with pentasodium diethylenetriamine pentaacetate compared with sodium chloride, significant improvement was observed from functional anosmia to hyposmia with a decrease of calcium cation concentration. Based on the results of this study, topical pentasodium diethylenetriamine pentaacetate may be a useful specific therapy for anosmia associated with COVD-19 infection.

\section{References}

1. Fokkens W, Landis B, Hopkins C, Reitsma S, Sedaghat A. Rhinology in review: from COVID-19 to biologicals. Rhinology. 2021.

2. Babaei A, Iravani K, Malekpour B, Golkhar B, Soltaniesmaeili A, Hosseinialhashemi M. Factors associated with anosmia recovery rate in COVID-19 patients. Laryngoscope Investigative Otolaryngology.2021;6(6):1248-1255.

3. Gallo O, Locatello LG, Mazzoni A, Novelli L, Annunziato F. The central role of the nasal microenvironment in the transmission, modulation, and clinical progression of SARS-CoV-2 infection. Mucosal immunology. 2021;14(2):305-316.

4. Whitcroft K, Ezzat M, Cuevas M, Andrews P, Hummel T. The effect of intranasal sodium citrate on olfaction in post-infectious loss: results from a prospective, placebo-controlled trial in 49 patients. Clinical 
Otolaryngology. 2017;42(3):557-563.

5. Whitcroft K, Gunder N, Cuevas M, et al. Intranasal sodium citrate in quantitative and qualitative olfactory dysfunction: results from a prospective, controlled trial of prolonged use in 60 patients.European Archives of Oto-Rhino-Laryngology. 2021:1-7.

6. Hart JR. Ethylenediaminetetraacetic acid and related chelating agents. Ullmann's encyclopedia of industrial chemistry. 2000.

7. Benes DM, Burnett CL. Final report on the safety assessment of pentasodium pentetate and pentetic acid as used in cosmetics.International journal of toxicology. 2008;27:71-92.

8. Sniffin'Sticks HT. Olfactory Performance Assessed by the Combined Testing of Odor Identification, Odor Discrimination and Olfactory Threshold. Chemical Senses. 1997;22(1):39-52.

9. Burke W. The ionic composition of nasal fluid and its function. Health. 2014;2014.

10. Švancara I, Vytřas K, Kalcher K, Walcarius A, Wang J. Carbon paste electrodes in facts, numbers, and notes: a review on the occasion of the 50-years jubilee of carbon paste in electrochemistry and electroanalysis. Electroanalysis: An International Journal Devoted to Fundamental and Practical Aspects of Electroanalysis.2009;21(1):7-28.

11.Vaira LA, Salzano G, Deiana G, De Riu G. Anosmia and ageusia: common findings in COVID-19 patients. The Laryngoscope.2020;130(7):1787-1787.

12. Gorzkowski V, Bevilacqua S, Charmillon A, et al. Evolution of olfactory disorders in COVID-19 patients. The Laryngoscope.2020;130(11):2667-2673.

13. Saniasiaya J, Islam MA, Abdullah B. Prevalence of Olfactory Dysfunction in Coronavirus Disease 2019 (COVID-19): A Meta-analysis of 27,492 Patients. The Laryngoscope. 2021;131(4):865-878.

14. Ronnett GV, Moon C. G proteins and olfactory signal transduction. Annual review of physiology. 2002;64(1):189-222.

15. Hummel T, Kobal G, Gudziol H, Mackay-Sim A. Normative data for the "Sniffin'Sticks" including tests of odor identification, odor discrimination, and olfactory thresholds: an upgrade based on a group of more than 3,000 subjects. European Archives of Oto-Rhino-Laryngology. 2007;264(3):237-243.

16. Rumeau C, Nguyen D, Jankowski R. How to assess olfactory performance with the Sniffin'Sticks test@. European Annals of Otorhinolaryngology, Head and Neck Diseases. 2016;133(3):203-206.

Table 1: Patient's characteristics

\begin{tabular}{llll}
\hline Character & Sodium chloride & DTPA & $\begin{array}{l}p \text { (Fisher exact } \\
\text { probability test) }\end{array}$ \\
\hline $\begin{array}{l}\text { Sample size, } \mathrm{n} \\
\text { Age (years), mean } \pm\end{array}$ & 33 & 33 & \\
$\begin{array}{l}\text { SD } \\
\begin{array}{l}\text { Days since symptoms } \\
\text { to enrollment, mean } \pm\end{array}\end{array}$ & $95 \pm 5.68$ & $38.25 \pm 7.62$ & \\
SD & & $98 \pm 6.27$ & \\
$\begin{array}{l}\text { Gender (male/female), } \\
\mathrm{n}\end{array}$ & $13 / 20$ & & \\
$\begin{array}{l}\text { Smokers } \\
\text { (current/never), n }\end{array}$ & $5 / 28$ & $15 / 18$ & \\
$\begin{array}{l}\text { Comorbidities, } \mathrm{n} \\
\text { Asthma }\end{array}$ & & \\
Diabetes & 2 & $4 / 29$ & 1.00 \\
& 7 & & \\
& & 3 & 1.00 \\
& 8 & 1.00
\end{tabular}




\begin{tabular}{|c|c|c|c|}
\hline Character & Sodium chloride & DTPA & $\begin{array}{l}p \text { (Fisher exact } \\
\text { probability test) }\end{array}$ \\
\hline Hypertension & 7 & 8 & 1.00 \\
\hline Migraine & 2 & 4 & 0.67 \\
\hline Sinusitis & 3 & 2 & 1.00 \\
\hline \multirow[t]{4}{*}{ Current medication } & Anti-histamine & Anti- histamine Glipizide & \\
\hline & Metformin B- blocker & Amlodipine ACE & \\
\hline & ACE inhibitor & inhibitor Paracetamol & \\
\hline & Sumatriptan Paracetamol & & \\
\hline \multirow[t]{2}{*}{$\begin{array}{l}\text { Previous anosmia } \\
\text { treatment }\end{array}$} & $\begin{array}{l}\text { None }(n=12) \text { Topical } \\
\text { steroids }(n=10)\end{array}$ & $\begin{array}{l}\text { None }(n=14) \text { Topical } \\
\text { steroids }(n=9)\end{array}$ & \\
\hline & $\begin{array}{l}\text { Systemic }+ \text { topical } \\
\text { steroids }(\mathrm{n}=11)\end{array}$ & $\begin{array}{l}\text { Systemic }+ \text { topical } \\
\text { steroids }(\mathrm{n}=7) \text { Vitamin } \\
\text { A }(\mathrm{n}=3)\end{array}$ & \\
\hline
\end{tabular}

Table 2. Results and statistical assessment before and after topical sodium chloride and DTPA

\begin{tabular}{lllll}
\hline & Sodium chloride & Sodium chloride & DTPA & DTPA \\
\hline & Pre administration & Post administration & Pre administration & Post a \\
T score, mean \pm SD & $2.48 \pm 0.56$ & $2.45 \pm 0.71$ & $2.54 \pm 0.38$ & $4.25 \pm 0$ \\
D score, mean \pm SD & $4.03 \pm 1.25$ & $4.05 \pm 0.92$ & $4.12 \pm 0.75$ & $7.28 \pm 0$ \\
I score, mean \pm SD & $4.39 \pm 0.88$ & $4.43 \pm 0.75$ & $5.09 \pm 1.08$ & $8.86 \pm 0$ \\
TDI score, mean \pm SD & $10.87 \pm 1.24$ & $10.93 \pm 1.09$ & $11.75 \pm 1.22$ & $20.39 \pm$ \\
Calcium cation concentration $(\mathrm{mM})$, mean $\pm \mathrm{SD}$ & $38.39 \pm 3.04$ & $37.06 \pm 2.64$ & $37.54 \pm 2.06$ & $24.39 \pm$ \\
\hline
\end{tabular}

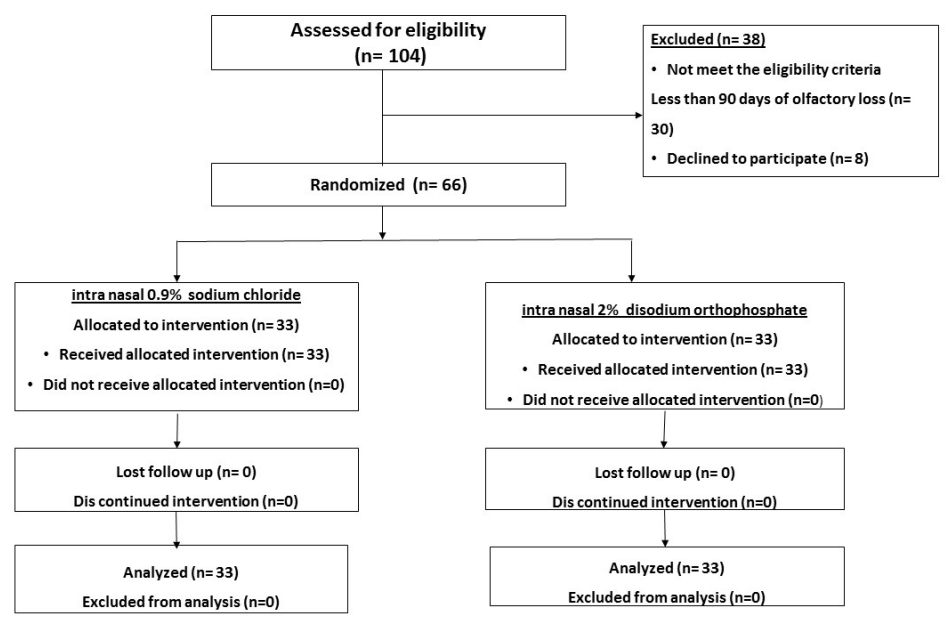

Figure1. The study design in accordance with reporting guidelines 


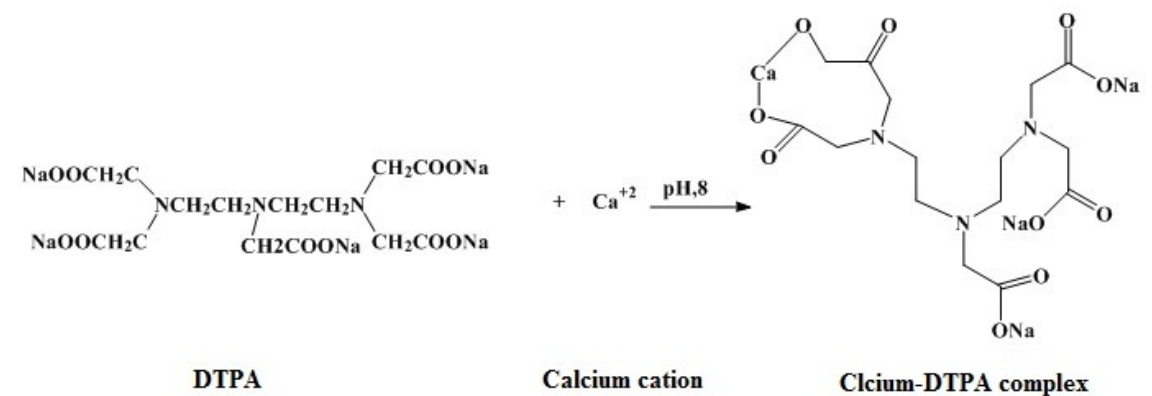

Figure 2. Schematic chemical reaction of calcium cation with DTPA 